\title{
Determination of the optimal set of frequency bands for the PLANCK CMBR satellite mission
}

\author{
M.J.D. Linden-Vørnle ${ }^{1}$ and H.U. Nørgaard-Nielsen ${ }^{2}$ \\ 1 Niels Bohr Institute for Astronomy, Physics and Geophysics, Astronomical Observatory, Juliane Maries Vej 30 , DK-2100 \\ København $\varnothing$, Denmark \\ 2 Danish Space Research Institute, Juliane Maries Vej 30, DK-2100 København Ø, Denmark
}

Received August 2, 1996; accepted July 1, 1997

\begin{abstract}
The PLANCK satellite (previously known as COBRAS/SAMBA) is chosen to be the next medium sized mission in ESAs Horizon 2000 programme. It is intended to observe anisotropies in the cosmic microwave background radiation (CMBR) with a sensitivity and angular resolution which is far better than previous CMBR experiments like NASAs COBE satellite mission. In order to assess PLANCKs ability to retrieve the temperature fluctuations in the presence of contaminating foreground sources and noise, we have performed model calculations based on a single-pixel approach and a non-linear least squares spectral fitting technique.

We find that even worst-case foreground scenarios do not prohibit a measurement accuracy of $\frac{\Delta T}{T} \sim 10^{-6}$. This implies that the above stated accuracy can be achieved over most of the sky and not only in selected areas with low foreground contamination as discussed by Brandt et al. (1994).

We have applied the model calculations to different mission configurations in order to determine the optimal combination of frequency bands. It is shown that only a large frequency coverage allows a sufficiently accurate separation of CMBR anisotropies and foreground signals. Also the Microwave Anisotropy Probe (MAP) CMBR mission, selected as one of the NASA MIDEX missions, is tested for its ability to overcome foreground contamination.
\end{abstract}

Key words: cosmic microwave background - ISM: general - space vehicles - methods: data analysis

\section{Introduction}

NASAs Cosmic Background Explorer (COBE) satellite mission has provided a wealth of information about the

Send offprint requests to: M.J.D. Linden-Vørnle
CMBR. The Far Infrared Spectrophotometer (FIRAS) has established the shape of the CMBR spectrum as an almost perfect black-body corresponding to a temperature of 2.728 \pm 0.004 (Fixsen et al. 1996) and the complete fouryear data from the Differential Microwave Radiometer (DMR) experiment demonstrate frequency independent RMS temperature fluctuations of the CMBR with a typical amplitude of $\sim 35 \pm 2 \mu \mathrm{K}$ on a $7^{\circ}$ angular scale (Banday et al. 1997). The fact that the fluctuations are frequency independent is consistent with the hypothesis, that they are of cosmological origin (Banday et al. 1997).

These important results along with power spectrum analysis of the four-year COBE anisotropy maps (see e.g. Bennett et al. 1996; Tegmark 1996; Górski et al. 1996) are however not able to impose sufficient constraints on theoretical models for the early Universe. The main objective for the second generation of CMBR experiments will thus be to make observations which can allow an unambiguous discrimination between competing models for the physics of the very early Universe as well as the formation and evolution of large-scale structure in the Universe. In order to achieve these goals, sensitivities of $\frac{\Delta T}{T} \sim 10^{-6}$ down to a $10^{\prime}$ angular scale covering at least one third of the sky will have to be reached (Bersanelli et al. 1996). Since the $10^{\prime}$ angular scale is smaller than that subtended by the horizon scale at the surface of last scattering and measurements made at the $\frac{\Delta T}{T} \sim 10^{-6}$ level will be limited only by cosmic variance, such observations will not only be a significant improvement with respect to the COBE results but will actually be the definitive observations of the CMBR anisotropies.

For the first generation of CMBR experiments lack of sufficient instrument sensitivity has been a major obstacle to reaching the above mentioned levels of sensitivity. In recent years developments in detector technology now make feasible the construction of CMBR experiments with instrumental sensitivities for $\frac{\Delta T}{T}$ in the order of a few times $10^{-6}$ (Bersanelli et al. 1996). With these instrument sensitivities the dominant remaining obstacle for high 
sensitivity anisotropy measurements is contamination by galactic and extragalactic foregrounds. For any experiment aiming at the detection of the extremely weak temperature fluctuations in the CMBR it is thus imperative to document how well this contamination can be overcome. The choice of optimal frequency bands enters this discussion as a crucial parameter.

The COBE FIRAS and DMR results along with observations of the infrared (IR) and far infrared (FIR) emission from our Galaxy made by the COBE FIRAS and Diffuse Infrared Background Experiment (DIRBE) has shown that space-based CMBR experiments with full or near full sky coverage have many advantages over groundbased or balloon-borne experiments. Away from atmospheric emission in a thermally stable environment the possibility for low-noise, multifrequency observation dramatically improves the ability to identify and remove hampering foreground effects of galactic and extragalactic nature. This ability is based on the difference in spectral and spatial signatures of the CMBR and the various foregrounds.

Various studies of this issue have been made including the work of Brandt et al. (1994) and Tegmark \& Efstathiou (1996). Brandt et al. (1994) identify two $10^{\circ} \times$ $10^{\circ}$ patches in the sky with the lowest possible foreground fluctuation levels, and only carry out their modelling with the limited foreground parameter ranges given in these patches. They investigate a number of different idealized experiments with a varying level of noise to establish at what noise level the anisotropy recovery becomes too difficult or impossible. The analysis is based on a single-pixel non-linear least-squares spectral fitting technique. They investigate the performance of both ground- and spacebased experiments in various frequency intervals and with various approaches to the foreground modelling depending on the frequency interval used (i.e. disregarding the effect of certain foregrounds in some frequency intervals). Their work indicates that the ablility of the spectral fitting technique to extract the CMBR anisotropies depends strongly on the noise level, which in turn depends in a highly nonlinear way on the frequencies observed. Obviously, the number of frequency bands i.e. data points must exceed the number of free parameters in the fit. If a given frequency range is divided into $N$ bands, then the extraction of anisotropies only weakly depends on $N$ as long as the former condition is met. They also conclude that a larger frequency coverage results in a better anisotropy extraction, but note that extending the frequency range also can have the opposite effect. If the frequency coverage is extended into regions where one or more foreground components which gave negligible contributions in the previous range become important, the amount of data needed to constrain the fit can increase considerably. Brandt et al. (1994) indicate that the reason for this is the highly nonlinear character of the extraction process. Attempts to remove effects from an inadequately constrained foreground component using this method can introduce errors many times larger than the fluctuations in the component itself.

Tegmark \& Efstathiou (1996) describe a different approach towards the removal of foreground effects from multi-frequency CMBR sky maps. They use a generalized Wiener filtering method to recover CMBR anisotropies in the presence of contaminating foregrounds from multifrequency data where the subtraction of foreground templates is optimized in the multipole-frequency $(\ell-\nu)$ plane in Fourier space. This method exploits the fact that the foregrounds have both spectral behaviour and angular power spectra which differ substantially from that of the CMBR.

This very persuasive scheme however has a major weakness, by being very dependent on the knowledge of the foregrounds, since the highly non-linear spectral behaviour of the foregrounds is treated as a linear problem. If the frequency dependencies of the foregrounds were perfectly known and independent of the position on the sky, this optimal Wiener filtering method could recover the CMBR anisotropy power spectrum from observations with $10^{\prime}$ angular resolution corresponding to multipoles of about $\ell \sim 1000$ with an accuracy of about one tenth of a percent (Tegmark \& Efstathiou 1996).

Since, however, the foreground parameters are rather uncertain, and since they are definitely not independent of the position in the sky (see discussion of the different foregrounds below), the accuracy stated above is not directly achievable.

In this work the non-linear single-pixel spectral fitting approach used by Brandt et al. (1994) is applied but we do not restrict ourselves to the limited range of foreground parameters found in selected areas with the lowest possible foreground emission as did Brandt et al. (1994). Instead we apply the method with extreme values for the foreground parameters i.e. modelling almost the entire sky. Furthermore we investigate the effect of our limited knowledge of the foregrounds by fitting functions which differ substantially from those used to create the simulated observations. Thus the ability to recover the CMBR anisotropies in the presence of poorly known foregrounds which can be encountered in most of the sky is tested for the PLANCK CMBR mission, which is selected to be ESAs next medium sized satellite mission. The ability to overcome the difficulties induced by foregrounds is discussed for the PLANCK baseline as well as for alternate configurations in order to assess the question of the optimum set of frequency bands and detector systems. Also the Microwave Anisotropy Probe (MAP), selected as one of the NASA MIDEX missions, is modelled and discussed.

\section{The PLANCK and MAP missions}

The main characteristics for the PLANCK satellite mission as given by Bersanelli et al. (1996) is a spin stabilized spacecraft with a payload consisting mainly of a telescope, 
Table 1. The main characteristics for the PLANCK baseline payload. The sensitivities given as intensity are in units of $10^{-20} \mathrm{erg} \mathrm{s}^{-1} \mathrm{~cm}^{-2} \mathrm{~Hz}^{-1} \mathrm{sr}^{-1}$ (Linden-Vørnle 1995)

\begin{tabular}{|l|c|c|c|c|c|c|c|c|c|}
\hline Instrument & \multicolumn{7}{|c|}{ Low Frequency $(\mathrm{LFI})$} & \multicolumn{7}{|c|}{ High Frequency (HFI) } \\
\hline \hline Center frequency, $\nu_{\mathrm{c}}[\mathrm{GHz}]$ & 31.5 & 53 & 90 & 125 & 150 & 217 & 353 & 545 & 857 \\
\hline Detector type & \multicolumn{3}{|c|}{ HEMT Radiometers } & \multicolumn{7}{|c|}{ Bolometers } \\
\hline Number of detectors & 4 & 14 & 26 & 12 & 8 & 12 & 12 & 12 & 12 \\
\hline Angular resolution $[$ arcmin] & 30 & 18 & 12 & 12 & 10.3 & 7.1 & 4.4 & 4.4 & 4.4 \\
\hline Bandwidth $\left(\Delta \nu / \nu_{\mathrm{c}}\right)$ & 0.15 & 0.15 & 0.15 & 0.15 & 0.37 & 0.37 & 0.37 & 0.37 & 0.37 \\
\hline \multicolumn{1}{|c|}{$\sigma$ sensitivities } & per pix for a 2 yr mission with a $30^{\prime}$ beam \\
\hline$\Delta T / T$ (Thermodynamic) $\left[10^{-6}\right]$ & 5.9 & 3.4 & 4.4 & 10.7 & 0.3 & 0.4 & 1.3 & 8.4 & 465.3 \\
\hline Intensity & 0.48 & 0.75 & 2.43 & 9.52 & 0.33 & 0.48 & 1.07 & 1.31 & 1.83 \\
\hline
\end{tabular}

a focal plane assembly and passive and active cooling devices. The telescope is a $1.5 \mathrm{~m}$ off-axis Gregorian system with the line-of-sight inclined $70^{\circ}$ with respect to the spin axis. The main characteristics for the payload are listed in Table 1. As can be seen from Table 1, the instrumental sensitivities of the frequency bands centered on the peak of the CMBR are in the order of $\frac{\Delta T}{T} \sim 10^{-6}$. This is also indicated in Figs. 1 and 2.

The MAP mission as described by Spergel et al. (1996) is also a spin stabilized spacecraft with a payload consisting of a dual back-to-back optical system providing COBE-like differential symmetry and passively cooled HEMT based detectors. The two telescopes are also $1.5 \mathrm{~m}$ off-axis Gregorian systems with the line-of-sight inclined $67.5^{\circ}$ with respect to the spin axis. MAP is expected to reach a $\frac{\Delta T}{T}$ sensitivity of $\sim 710^{-6}$ per $0.3 \times 0.3$ degree $^{2}$ pixel (Spergel et al. 1996) so it is clear, that it is not the intention for MAP to reach the same sensitivity as the PLANCK mission. An overview of the main payload characteristics is given in Table 2 .

\section{Contaminating foreground sources}

As mentioned earlier, the development of detector technology is advancing rapidly, producing detectors for CMBR experiments with very low noiselevels as indicated in Tables 1 and 2. Thus, the main obstacle for at least space based experiments is the contribution from different galactic and extragalactic foreground sources to the emission in the microwave and FIR sky.

The galactic foregrounds are identified as:

- Synchrotron radiation.

- Free-free emission.

- Emission from galactic dust.

The extragalactic sources mainly consist of:

- Point sources (radio and infrared).

- Sunyaev-Zel'dovich effect from clusters of galaxies.

Solar system objects like the Sun, the Earth and the Moon also play a vital role as effects contaminating observations of the CMBR anisotropies introducing noise by increased level of radiation. Unlike for the galactic and extragalactic sources, it is possible to compensate strongly for the effects introduced by solar system objects by choosing an orbit which minimizes these effects. It is shown by Muciaccia et al. (1996), that placing a satellite like PLANCK in the Lagrangian L2 point of the Sun/Earth system makes the effect induced by the Sun, the Earth and the Moon possible to handle. When the satellite points in anti-solar direction these objects will always be at large angles making straylight rejection and thermal control possible in order to achieve the high sensitivity required.

Only Jupiter will enter the field of view resulting in some loss of sky coverage. The planet can and will on the other hand be used for calibration purposes.

The effect of extragalactic sources on the observation of CMBR anisotropies has been studied extensively by e.g. Franceschini et al. (1989). Their results show, that point source confusion mainly becomes a problem on the $\frac{\Delta T}{T} \sim 10^{-6}$ level at angular resolutions better than $30^{\prime}$ at least for the 50 to $200 \mathrm{GHz}$ range. At higher frequencies IR point sources become increasingly troublesome.

With regards to the diffuse galactic emission Tegmark $\&$ Efstathiou (1996) find that the fluctuation power spectrum of these foregrounds is roughly consistent with $C_{\ell} \propto$ $(5+\ell)^{-3}$ assuming a coherence scale of $\ell \approx 10$ corresponding to about $10^{\circ}$. This implies that fluctuations in the galactic foregrounds will be of less importance for higher $\ell$ i.e. smaller angular scales.

In the following a discussion of the foregrounds included in the modelling in this work is given. These include only galactic sources since the modelling is done only for a $30^{\prime}$ angular resolution for all frequency bands for the PLANCK mission and parts thereof and for an $18^{\prime}$ resolution for the MAP mission i.e. at angular scales where fluctuations from extragalactic sources are largely negligible.

\subsection{Synchrotron radiation}

The galactic synchrotron radiation is produced by Cosmic Ray (CR) electrons spiraling in the magnetic field of the 
Table 2. The main characteristics for the MAP baseline payload adopted from Spergel et al. (1996). We have calculated the sensitivity in $\Delta T / T$ and intensity assuming a noise temperature, $\Delta T_{\mathrm{A}}$, of $25 \mu \mathrm{K}$ per $0.3 \times 0.3$ degree ${ }^{2}$ pixel per frequency band. The sensitivties given as intensity are in units of $10^{-20} \mathrm{erg} \mathrm{s}^{-1} \mathrm{~cm}^{-2} \mathrm{~Hz}^{-1} \mathrm{sr}^{-1}$

\begin{tabular}{|l|c|c|c|c|c|}
\hline Center frequency, $\nu_{\mathrm{c}}[\mathrm{GHz}]$ & 22 & 30 & 40 & 60 & 90 \\
\hline Detector type & \multicolumn{5}{|c|}{ HEMT Radiometers } \\
\hline Number of detectors & 4 & 4 & 8 & 8 & 16 \\
\hline Angular resolution $[$ arcmin] & 54 & 39 & 31.8 & 23.4 & 17.4 \\
\hline Bandwidth $\left(\Delta \nu / \nu_{\mathrm{c}}\right)$ & 0.18 & 0.17 & 0.28 & 0.18 & 0.19 \\
\hline $1 \sigma$ sensitivities per pixel for a 1 yr mission with a $18^{\prime}$ beam \\
\hline$\Delta T / T$ (Thermodynamic) $\left[10^{-6}\right]$ & 9.3 & 9.4 & 9.6 & 10.1 & 11.3 \\
\hline Intensity & 0.37 & 0.69 & 1.23 & 2.77 & 6.23 \\
\hline
\end{tabular}

Galaxy. It can be shown, that the emissivity and thus the intensity of the synchrotron radiation as a function of frequency follows a power-law if the energy spectrum for the CR electrons is assumed also to follow a power-law. The latter has been empirically established by means of observations of CR electrons close to Earth (e.g. Salter \& Brown 1988). The intensity of the galactic synchrotron radiation can thus be expressed as:

$I_{\text {Sync }}(\nu) \propto \nu^{-\alpha}$.

Here $\alpha=(\delta-1) / 2$, where $\delta$ is the spectral index for the CR electron energy spectrum.

Radio surveys made at $408 \mathrm{MHz}$ covering the entire sky with an angular resolution of $51^{\prime}$ (Haslam et al. 1982) and at $1420 \mathrm{MHz}$ covering the northern hemisphere down to declination $-19^{\circ}$ with a $34^{\prime}$ resolution (Reich 1982 and Reich \& Reich 1986) show, that there is a rather significant variation of the spectral index. A variation of $\alpha$ from 1.3 to 0.1 with a mean of 0.7 at declinations spanning from $+20^{\circ}$ to $+90^{\circ}$ was found by Brandt et al. (1994) by fitting spatially corresponding pixels of the two surveys and then fitting to a power-law.

But not only does the spectral index vary with position - it also tends to steepen with increasing frequency (Lawson et al. 1987). This steepening is thought to be induced by diffusive shock acceleration of the CR electrons perhaps caused by supernova explosions.

The overall picture is thus very complicated. The radiation depends on several parameters like electron density, electron energy distribution and magnetic field strength, which are not well known or rather not known for every position in the Galaxy. The only way to make significant progress in the attempts to describe the galactic synchrotron radiation is, by making high sensitivity, multifrequency measurements covering the entire sky with constant, high angular resolution and beam pattern. Such work by others is in progress (Danese et al. 1996) and should soon yield results.

The PLANCK mission will however produce high quality maps of the entire sky at frequencies where synchrotron radiation dominates. Such maps will be of great importance for constraining models for this radiation source. It will also make PLANCK more independent of groundbased observations and will thus play a significant role in the actual data processing.

In the present study, the synchrotron radiation is modelled as a perfect power-law, where both the spectral index and the baselevel are allowed to vary independently within limits, which should be sufficient to model any local variation. The spectral indices used in the models are thus not assumed to be frequency dependent.

\subsection{Free-free emission}

The galactic free-free emission originates from free electrons in the approximately $10^{5} \mathrm{~K}$ hot ionized interstellar medium (ISM), which are deflected and thus slowed down by ionized atoms, mostly protons. The difference in kinetic energy of the electron before and after the deflection is the amount of energy radiated as free-free emission or "bremsstrahlung".

Bennett et al. (1992) derive an expression giving the antenna or Rayleigh-Jeans temperature induced by the free-free emission as a function of frequency and find that the frequency dependence of the antenna temperature follows a power-law, $T_{\mathrm{A}} \propto \nu^{-\alpha}$. The spectral index $\alpha$ is a weak function of frequency and the kinetic electron temperature. In terms of intensity the frequency dependence of the free-free emission also takes the shape of a powerlaw with a spectral index of $\sim 0.17$.

The free-free emission component of our Galaxy is still rather poorly understood in the sense, that no usable allsky maps exist. Bennett et al. (1992) produced all-sky maps from the COBE DMR data using both a subtraction and a fitting technique. Both maps are noise dominated - especially on small scales - and model dependent, since the modelling of the synchrotron radiation is uncertain (Brandt et al. 1994). Some conclusions can nevertheless be drawn from these maps, namely that the galactic freefree emission almost follows a cosecant-law when going to higher galactic latitudes (Bennett et al. 1992). The maps 
also show that the bulk of galactic free-free emission is confined in the plane of the Galaxy. This should come as no surprise since the hot, massive stars, which produce the environment suitable for making free-free emission are all located in the galactic plane (Partridge 1995).

Besides this work by Bennett et al. (1992) there is another approach based on a relation between the intensity of $\mathrm{H} \alpha$ and free-free emission as discussed by Reynolds (1992). Unfortunately no full sky survey of the interstellar $\mathrm{H} \alpha$ background exists yet so this method can only be used as an further estimate of the distribution of the free-free emission. Such estimates confirm the cosecant-law found by Bennett et al. (1992).

Kogut et al. (1995) have reported a spatial correlation between free-free emission and galactic dust emission (see below) on angular scales larger than the $7^{\circ} \mathrm{COBE}$ DMR beam. They conclude that if this correlation holds to smaller angular scales, free-free emission should be a less significant contaminant for anisotropy measurements on degree scales at frequencies above $20 \mathrm{GHz}$.

This is confirmed by recent work by Simonetti et al. (1996), which indicates that free-free emission contribution to fluctuations at $27.5 \mathrm{GHz}$ are negligible to $\mathrm{CMBR}$ anisotropies on degree-scales. These results were obtained by making sensitive $\mathrm{H} \alpha$ images and comparing these with recent data from the Saskatoon CMBR experiment.

Even though the lack of surveys of the galactic freefree emission leaves many open questions, the situation is a bit more relaxed than with respect to the synchrotron radiation since the possible range of parameters, like spectral index, are more constrained, as the physical processes causing the free-free emission are less sensitive to local changes in the environment (e.g. kinetic electron temperature).

In this work the free-free emission is modelled as a perfect power-law, as is the synchrotron radiation, where both parameters are allowed to vary within reasonable limits, in order to cover the resulting emission range produced by local variations.

\subsection{Galactic dust emission}

The galactic dust consists of conglomerates of molecules in a range of sizes from $\sim 5 \AA$ to $30 \mu \mathrm{m}$, of which most are formed in the "cool" atmospheres of red giant stars and from there ejected into the ISM. Here the dust grains can be heated by the photon flux from stars and will then seek thermodynamic equilibrium by emitting radiation in the IR or the FIR part of the spectrum (see e.g. Alexander \& Ferguson 1994; Sedlmayr 1994; Knapp 1991).

Dust will radiate as a grey-body, meaning that the emission spectrum for the dust will be a black-body corresponding to the equilibrium temperature, $T$, of the dust modified with a frequency dependent emissivity conventionally given as $K \nu^{\alpha}$ (Banday \& Wolfendale 1991). The intensity of dust emission from a population of dust grains heated to the temperature, $T$, would then be:

$I_{\text {Dust }}(\nu)=K \nu^{\alpha} \times B_{\nu}(T)$

where $B_{\nu}(T)$ is the black-body radiation corresponding to the temperature, $T$. The spectral index, $\alpha$, depends on the atomic and geometric structure and composition of the dust grains (Banday \& Wolfendale 1991). Detailed calculations and laboratory experiments have demonstrated that $\alpha \approx 2$ for larger grains, whilst smaller grains tend to index values lower than 2 .

Due to the contaminating effect of the Earth atmosphere, most information about the galactic dust emission is supplied by observations made by satellites i.e. IRAS and COBE.

Especially the COBE FIRAS and DIRBE instruments have provided vast amounts of information about the galactic dust at IR and FIR wavelength. Wright et al. (1991) reported preliminary spectral observations of the Galaxy by FIRAS, stating that there are two major components in the spectrum of the Galaxy, namely a continuous component due to heated dust and strong line emission from the $158 \mu \mathrm{m}$ line of singly ionized carbon.

With respect to the continuum, Wright et al. (1991) split the dust emission into two functions - one of position (i.e. galactic longitude and latitude) and one describing the spectral behaviour $(g(\nu))$. In order to determine the spectral behaviour Wright et al. (1991) tried out different forms of analytic fits with functions of the form given by Eq. (2). They found that the best fit was obtained with the expression:

$g(\nu) \approx 0.00016\left(\frac{\nu}{900 \mathrm{GHz}}\right)^{1.65} B_{\nu}(23.3 \mathrm{~K})+S(\nu)$,

where the term $S(\nu)$ represents the galactic synchrotron radiation component. They obtained an even better fit, when introducing a cold dust component with a temperature of $4.77 \mathrm{~K}$ :

$$
\begin{aligned}
g(\nu) \approx & 0.00022\left(\frac{\nu}{900 \mathrm{GHz}}\right)^{2} \\
& \times\left[B_{\nu}(20.4 \mathrm{~K})+6.7 B_{\nu}(4.77 \mathrm{~K})\right]+S(\nu) .
\end{aligned}
$$

The better fit by the two-temperature dust model is due to the fact, that the FIRAS data exhibit an excess of emission at long wavelength. The $4.77 \mathrm{~K}$ black-body emission supplies the excess needed to fit the data.

Similar spectral fitting, also based on the FIRAS data, has been performed by Reach et al. (1995). They also find evidence for a widespread, very cold $(4-7 \mathrm{~K})$ dust component, which has an optical depth that is spatially correlated with the "warm" $(\sim 20 \mathrm{~K})$ dust. They argue that the cold component could either be a result of enhanced emissivity of the warm dust at submilimeter wavelength or be caused by a so far unknown population of dust grains. 
The subject is nevertheless controversial since Boulanger et al. (1996) find, that the emission excess which Wright et al. (1991) and Reach et al. (1995) interpret as a cold component is not correlated with the $21 \mathrm{~cm}$ emission which traces the neutral hydrogen. Boulanger et al. (1996) conclude that the excess is either due to an unknown extragalactic source or caused by the FIRAS instrument. The work of Kogut et al. (1995), which indicates a correlation between the galactic free-free and dust emission does not exclude the possibility of a cold dust component but does not support it either.

Results from the COBE DIRBE instrument are not able to settle this dispute since the longest wavelength utilized by DIRBE is $240 \mu \mathrm{m}$. Any dust component radiating at $5-10 \mathrm{~K}$ would not contribute significantly to the intensity at $240 \mu \mathrm{m}$ implying that such a component would remain largely undetected by DIRBE (Sodroski et al. 1994).

In this discussion one could find arguments to support the modelling of the galactic dust emission both as a two-temperature and a single-temperature dust. There is however a less complicated approach, which can be used. No matter whether the dust consists of one or more components, the Rayleigh-Jeans part of the dust spectrum can be approximated as a perfect power-law. Since the baseline frequency coverage of the PLANCK mission only just stretches further than the Rayleigh-Jeans part of the black-body for a $20 \mathrm{~K}$ dust component, the galactic dust emission is in this work modelled both as a twotemperature dust with a very cold component and as a perfect power-law with simultanious varying baselevel and spectral index covering all possible dust emission intensities demonstrated by the DIRBE $240 \mu \mathrm{m}$ map extrapolated to the wavelength range of PLANCK.

\section{Model calculations}

The model calculations in this study are, as mentioned earlier, inspired by the work of Brandt et al. (1994). The concept is to simulate differential observations of the CMBR with contaminating foreground sources on a singlepixel basis, to add realistic instrument noise and then try to recover the original model parameters by using a nonlinear least-squares spectral fitting technique.

By doing so, one should be able to address the very important issue of how well PLANCK and other mission concepts will be able to extract the anisotropies in the CMBR in the presence of hampering foregrounds.

A number of simplifying assumptions are made both with respect to the instrument, the simulated observations and in the actual fitting process. These assumptions, the models and the fitting are described below:

\subsection{The instrument}

- Frequency bands are assumed to have a zero bandwidth.
- For PLANCK and parts thereof noise (sensitivity) assumes a 2 year mission, an angular resolution of $30^{\prime}$ for all bands and only gaussian noise. See Table 1 for a listing of the assumed noise-levels (or sensitivities) in the frequency bands.

- For MAP noise (sensitivity) assumes a 1 year mission, an angular resolution of $18^{\prime}$ for all bands and only gaussian noise. See Table 2 for a listing of the assumed noise-levels (or sensitivities) in the frequency bands.

- Modelling is done on a single-pixel basis only regarding measurements in one resolution element (pixel) of the size $30 \times 30 \operatorname{arcmin}^{2}$ for PLANCK and $18 \times 18 \operatorname{arcmin}^{2}$ for MAP.

The fact that the modelling is done on a single pixel basis implies that any spatial correlation in the CMBR and in the foregrounds is ignored, which will make the modelling a conservative estimate.

\subsection{The simulated observations}

The following contaminating foregrounds are included in the simulated observations: Synchrotron radiation, freefree emission and galactic dust emission. The total model used to produce the simulated observations is given by:

$$
\begin{aligned}
\Delta I_{\nu, \text { model }}= & \Delta I_{\mathrm{CMBR}}+\Delta I_{\text {Synchrotron }}+\Delta I_{\text {Free }- \text { free }} \\
& +\Delta I_{\text {Dust }}+\Delta I_{\text {Noise }}
\end{aligned}
$$

where

$$
\begin{array}{ll}
\Delta I_{\mathrm{CMBR}} & =B_{\nu}(T)-B_{\nu}(\bar{T}) \\
\Delta I_{\text {Synchrotron }} & =I_{\text {sync }}\left(\frac{\nu}{\nu_{0}}\right)^{\alpha_{\text {sync }}}-\overline{I_{\text {sync }}}\left(\frac{\nu}{\nu_{0}}\right)^{\overline{\alpha_{\text {sync }}}} \\
\Delta I_{\text {Free-free }} & =I_{\text {free }}\left(\frac{\nu}{\nu_{0}}\right)^{\alpha_{\text {free }}}-\overline{I_{\text {free }}}\left(\frac{\nu}{\nu_{0}}\right)^{\alpha_{\text {free }}} \\
\Delta I_{\text {Dust }} & =I_{\text {dust }}\left(\frac{\nu}{\nu_{0}}\right)^{\alpha_{\text {dust }}}-\overline{I_{\text {dust }}}\left(\frac{\nu}{\nu_{0}}\right)^{\overline{\alpha_{\text {dust }}}} \\
\Delta I_{\text {Noise }} & =G \times I_{\text {Noise }} .
\end{array}
$$

Here $T=\bar{T}+\Delta T$, where $\bar{T}=2.728 \mathrm{~K}$ and $\Delta T$ is allowed to vary within $\pm 100 \mu \mathrm{K}$. $I_{\text {Noise }}$ is given in Table 1 and 2 as the $1 \sigma$ sensitivity in intensity and $G$ is a gaussian random variable yielding random values with $\langle G\rangle=0.0$

\begin{tabular}{|c|c|c|}
\hline $\bar{\alpha}_{\text {sync }}$ & $=$ & -0.93 \\
\hline$\overline{I_{\text {sync }}}$ & $=$ & 0.89 \\
\hline$\alpha_{\text {sync }}$ & $=$ & $\overline{\alpha_{\text {sync }}} \pm 0.35$ \\
\hline$I_{\text {sync }}$ & $=$ & $\overline{I_{\text {sync }}} \pm 0.27$ \\
\hline
\end{tabular}
and $1 \sigma=1.0$.

The parameters and parameter ranges for the foregrounds, originally adapted from Fig. 2 in Bennett et al. (1992), are given below with $\nu_{0}=100 \mathrm{GHz}$ and all intensities given in units of $10^{-20} \mathrm{erg} \mathrm{s}^{-1} \mathrm{~cm}^{-2} \mathrm{~Hz}^{-1} \mathrm{sr}^{-1}$.

Synchrotron radiation: 
Free-free emission:

\begin{tabular}{|llr|}
\hline$\overline{\alpha_{\text {free }}}=$ & -0.12 \\
$I_{\text {free }}$ & $=$ & 2.11 \\
$\alpha_{\text {free }}$ & $=$ & $\overline{\alpha_{\text {free }}} \pm 0.10$ \\
$I_{\text {free }}$ & $=$ & $\overline{I_{\text {free }}} \pm 1.16$ \\
\hline
\end{tabular}

Dust emission:

\begin{tabular}{|llr|}
$\overline{\alpha_{\text {dust }}}$ & $=$ & 3.43 \\
$\overline{I_{\text {dust }}}$ & $=$ & 3.72 \\
$\alpha_{\text {dust }}$ & $=$ & $\overline{\alpha_{\text {dust }}} \pm 1.60$ \\
$I_{\text {dust }}$ & $=$ & $\overline{I_{\text {dust }}} \pm 2.60$ \\
\hline
\end{tabular}

When the two-temperature dust model is used, $\Delta I_{\text {Dust }}$ is given by:

$$
\begin{aligned}
\Delta I_{\text {Dust }}= & \left(\frac{\nu}{\nu_{0}}\right)^{2} \times \tau\left[B_{\nu}(18.0 \mathrm{~K})+7 B_{\nu}(4.0 \mathrm{~K})\right] \\
& -\left(\frac{\nu}{\nu_{0}}\right)^{2} \times \bar{\tau}\left[B_{\nu}(18.0 \mathrm{~K})+7 B_{\nu}(4.0 \mathrm{~K})\right] .
\end{aligned}
$$

In this model the parameter and range is given with $\nu_{0}=$ $900 \mathrm{GHz}$ by:

Dust emission:

\begin{tabular}{|rrr|}
\hline $\bar{\tau}$ & $=$ & 0.3 \\
$\tau$ & $=$ & $\bar{\tau}_{-0.2}^{+0.7}$ \\
\hline
\end{tabular}

The allowed ranges of foregrounds are sketched in Fig. 1. These ranges of model parameters yield sky brightness contributions corresponding to situations which can be encountered in most of the sky. The exact fractions are: $99.6 \%$ of the entire sky for the synchrotron radiation, $86 \%$ of the entire sky for the free-free emission and $100 \%$ of the entire sky for the power-law dust. The two-temperature dust parameters cover $100 \%$ of the areas at high galactic latitudes, where very cold dust is located according to Reach et al. (1995), corresponding to about $20 \%$ of the entire sky.

These estimates of the parameter coverage are made by using various all-sky maps and extrapolating the intensities produced by the parameter ranges given above to these maps thus estimating the sky coverage.

For the synchrotron radiation the Haslam et al. (1982) map was used and for the dust emission the DIRBE $240 \mu \mathrm{m}$ map used. Since no direct map of the galactic freefree emission exists the assessment of the free-free coverage was made using the relation between $\mathrm{H} \alpha$ and free-free emission discussed by Reynolds (1992) and Bennett et al. (1992).

For each model a set of parameters $\left(\Delta T, I_{\mathrm{sync}}, \alpha_{\mathrm{sync}}\right.$, $I_{\text {free }}, \alpha_{\text {free }}$ and either $I_{\text {dust }}$ and $\alpha_{\text {dust }}$ or $\tau$ depending on the dust model) is chosen randomly staying within the limits listed above and sketched in Fig. 1. Using Eq. (5) the observed differential intensity, $\Delta I_{\nu, \text { model }}$, is then calculated for each frequency band.
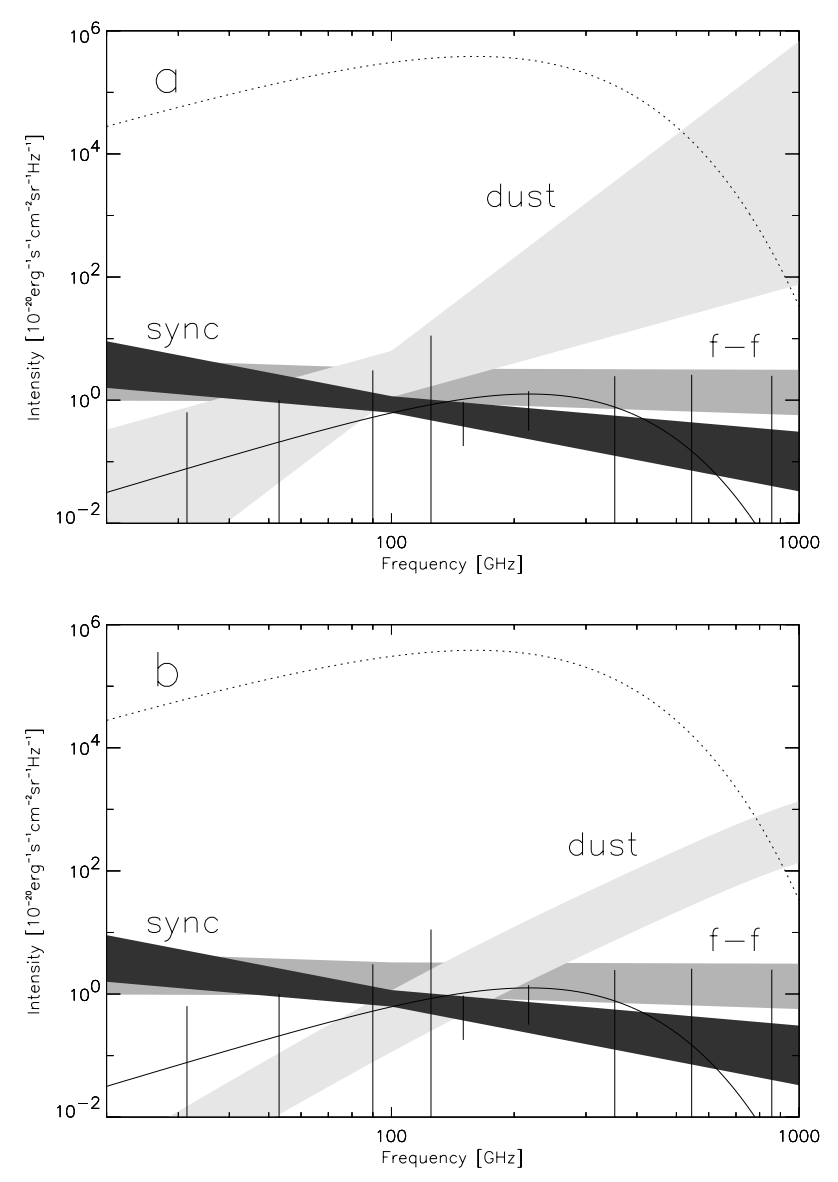

Fig. 1. The range of foregrounds given the parameter ranges covered by the model calculations. For both plots the dashed line is the $2.728 \mathrm{~K}$ black-body, the solid line corresponds to a $\frac{\Delta T}{T}$ of $10^{-6}$, the darkest grayshaded region is the synchrotron radiation (marked "sync"), the intermediate grayshaded region is the free-free emission (marked "f-f") and finally the lightest grayshaded region is the dust component (marked "dust"). The solid vertical lines illustrate PLANCK instrument noise levels given in Table 1. In a) the parameters for the simulations with the perfect power-law dust model are shown and in b) the ranges for the two-temperature dust simulations are displayed

\subsection{The parameter recovery process}

The recovery of the model parameters was performed by fitting a function similar to Eq. (5) to the simulated observations. The fitting was performed using the programme ADAPTION (Brosa 1994) for non-linear least squares fitting. This programme uses Gauss' method for least squares fitting as described by e.g. Press et al. (1989) whereas Brandt et al. (1994) use the LevenbergMarquardt method (also described by Press et al. 1989). As Brandt et al. (1994) we have taken great care to prevent local minima from contaminating our results. 


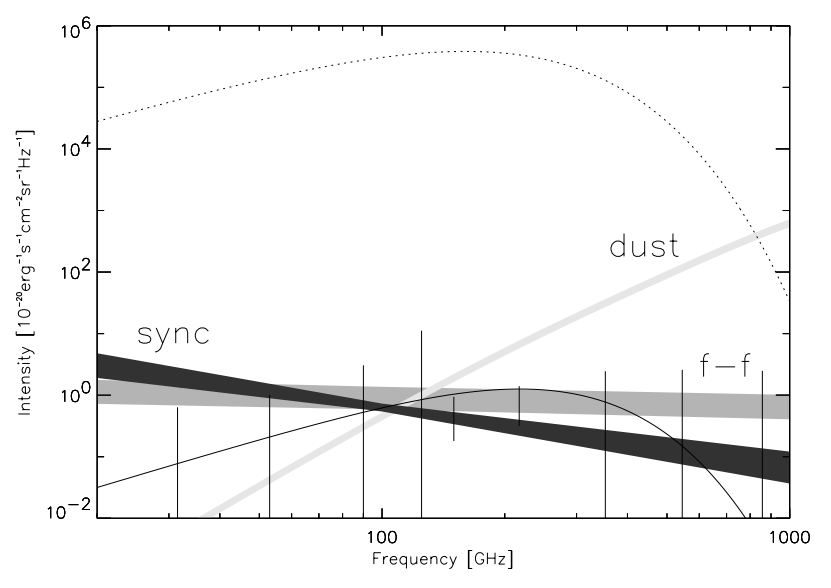

Fig. 2. The range of foregrounds used in the model calculations of Brandt et al. (1994). The dashed line is the $2.728 \mathrm{~K}$ black-body, the solid line corresponds to a $\frac{\Delta T}{T}$ of $10^{-6}$, the darkest grayshaded region is the synchrotron radiation (marked "sync"), the intermediate grayshaded region is the free-free emission (marked "f-f") and finally the lightest grayshaded region is the dust component (marked "dust"). As in Fig. 1 the PLANCK instrument noise levels are indicated by vertical lines

Important assumptions regarding the fitting are made:

- Combined synchrotron radiation and free-free emission are fitted using a single power-law.

- The power-law dust model is fitted with a power-law.

- The two-temperature component dust model is fitted with a two-temperature component model, but with the coldest dust temperature chosen $1 \mathrm{~K}$ too high (i.e. $5 \mathrm{~K}$ compared to $4 \mathrm{~K}$ in the simulated observations). The reason for introducing this temperature difference in the fitting of the cold dust component is to investigate the effect of our limited knowledge of such a component with a temperature close to that of the CMBR black-body.

The function which is fitted to the simulated observations is thus given by:

$\Delta I_{\nu, \text { fit }}=\Delta I_{\mathrm{CMBR}, \mathrm{fit}}+\Delta I_{\mathrm{Sync} / \mathrm{ff}, \mathrm{fit}}+\Delta I_{\text {Dust }, \mathrm{fit}}$

where

$$
\begin{aligned}
& \Delta I_{\mathrm{CMBR}, \mathrm{fit}}=B_{\nu}\left(\bar{T}+\Delta T_{\mathrm{fit}}\right)-B_{\nu}(\bar{T}) \\
& \Delta I_{\mathrm{Sync} / \mathrm{ff}, \mathrm{fit}}=I_{\mathrm{s} / \mathrm{f}, \mathrm{fit}}\left(\frac{\nu}{\nu_{0}}\right)^{\alpha_{\mathrm{s} / \mathrm{f}, \mathrm{fit}}}-\overline{I_{\mathrm{s} / \mathrm{f}}}\left(\frac{\nu}{\nu_{0}}\right)^{\overline{\alpha_{\mathrm{s} / \mathrm{f}}}} \\
& \Delta I_{\text {Dust,fit }}=I_{\text {dust,fit }}\left(\frac{\nu}{\nu_{0}}\right)^{\alpha_{\text {dust,fit }}}-\overline{I_{\text {dust }}}\left(\frac{\nu}{\nu_{0}}\right)^{\overline{\alpha_{\text {dust }}}}
\end{aligned}
$$

Here $\overline{I_{\mathrm{s} / \mathrm{f}}}=\overline{I_{\text {sync }}}+\overline{I_{\text {free }}}=3.00$ and $\overline{\alpha_{\mathrm{s} / \mathrm{f}}}=\frac{\left(\overline{\alpha_{\mathrm{sync}}}+\overline{\alpha_{\mathrm{free}}}\right)}{2}=$ -0.54 .

For the case where the dust is modelled with a twotemperature component model $\Delta I_{\text {Dust,fit }}$ is given by:

$$
\begin{aligned}
\Delta I_{\text {Dust }, \text { fit }}= & \left(\frac{\nu}{\nu_{0}}\right)^{2} \times \tau_{\text {fit }}\left[B_{\nu}(18.0 \mathrm{~K})+7 B_{\nu}(5.0 \mathrm{~K})\right] \\
& -\left(\frac{\nu}{\nu_{0}}\right)^{2} \times \bar{\tau}\left[B_{\nu}(18.0 \mathrm{~K})+7 B_{\nu}(5.0 \mathrm{~K})\right] .
\end{aligned}
$$

Note the $1 \mathrm{~K}$ difference in the coldest dust temperature between the simulated observations and the model fitted to the data.

In order to get a statistical sample 100 different models are calculated and the difference between the input parameters $\left(\Delta T,\left(I_{\text {sync }}+I_{\text {free }}\right), \frac{\left(\alpha_{\text {sync }}+\alpha_{\text {free }}\right)}{2}, I_{\text {dust }}\right.$ and $\alpha_{\text {dust }}$ or $\tau)$ and the fitted parameters $\left(\Delta \stackrel{2}{\text { fit }}, I_{\mathrm{s} / \mathrm{f}, \mathrm{fit}}, \alpha_{\mathrm{s} / \mathrm{f}, \text { fit }}\right.$ and $I_{\text {dust,fit }}$ and $\alpha_{\text {dust,fit }}$ or $\tau_{\text {fit }}$ ) are registered (e.g. $\Delta T-\Delta T_{\text {fit }}$ ). From these differences means and standard deviations are calculated.

\section{Results}

Below are the results of calculations for the standard PLANCK baseline and for the PLANCK LFI and HFI concepts separately.

Also the results for a revised PLANCK concept only including frequency bands $31.5,53,150,217,545$ and $857 \mathrm{GHz}$, a concept based only on bolometric detectors ${ }^{1}$ with frequency bands 53, 90, 150, 217, 353, 545 and 857 $\mathrm{GHz}$ and results for the MAP concept are presented. For each mission concept the frequency bands used are listed in $\mathrm{GHz}$ and the resulting means and standard deviations for $\Delta T-\Delta T_{\text {fit }}$ are given in units of $\mu \mathrm{K}$.

\section{PLANCK Baseline:}

\begin{tabular}{|l|c|c|}
\hline \multicolumn{3}{|c|}{ Bands [GHz]: 31.5 5390125 150 217 353 545 857 } \\
\hline Dust model: & $\left\langle\Delta T-\Delta T_{\text {fit }}\right\rangle[\mu \mathrm{K}]$ & $\sigma_{\Delta T-\Delta T_{\text {fit }}}[\mu \mathrm{K}]$ \\
\hline Power-law & 0.06 & 0.65 \\
\hline Two-temp. & 0.51 & 1.08 \\
\hline
\end{tabular}

\section{PLANCK Bolometer:}

\begin{tabular}{|l|c|c|}
\hline \multicolumn{3}{|c|}{ Bands [GHz]: 5390150217353545857} \\
\hline Dust model: & $\left\langle\Delta T-\Delta T_{\text {fit }}\right\rangle[\mu \mathrm{K}]$ & $\sigma_{\Delta T-\Delta T_{\text {fit }}}[\mu \mathrm{K}]$ \\
\hline Power-law & -0.07 & 0.77 \\
\hline Two-temp. & 0.44 & 0.97 \\
\hline
\end{tabular}

PLANCK Revised:

\begin{tabular}{|l|c|c|}
\hline \multicolumn{3}{|c|}{ Bands [GHz]: 31.5 53150217 545857} \\
\hline Dust model: & $\left\langle\Delta T-\Delta T_{\text {fit }}\right\rangle[\mu \mathrm{K}]$ & $\sigma_{\Delta T-\Delta T_{\text {fit }}}[\mu \mathrm{K}]$ \\
\hline Power-law & -0.03 & 0.82 \\
\hline Two-temp. & -0.24 & 1.13 \\
\hline
\end{tabular}

1 The $\frac{\Delta T}{T}$ sensitivities assumed for this concept are (in units of $10^{-6}: 0.4,0.4,0.3,0.4,1.3,8.4,465.3$. 
HFI:

\begin{tabular}{|l|c|c|}
\hline \multicolumn{3}{|c|}{ Bands $[\mathrm{GHz}]: 150217353545857$} \\
\hline Dust model: & $\left\langle\Delta T-\Delta T_{\text {fit }}\right\rangle[\mu \mathrm{K}]$ & $\sigma_{\Delta T-\Delta T_{\text {fit }}}[\mu \mathrm{K}]$ \\
\hline Power-law & -0.01 & 0.93 \\
\hline Two-temp. & 0.35 & 1.19 \\
\hline
\end{tabular}

LFI:

\begin{tabular}{|l|c|c|}
\hline \multicolumn{3}{|c|}{ Bands [GHz]: 31.5 53 90 125 } \\
\hline Dust model: & $\left\langle\Delta T-\Delta T_{\text {fit }}\right\rangle[\mu \mathrm{K}]$ & $\sigma_{\Delta T-\Delta T_{\text {fit }}}[\mu \mathrm{K}]$ \\
\hline Power-law & -11.88 & 33.38 \\
\hline Two-temp. & 1.96 & 36.37 \\
\hline
\end{tabular}

With respect to the other parameters only those related to the dust emission can be stated since the synchrotron radiation and free-free emission are fitted as one power-law. The results for the dust show, that the spectral indecies and base-levels or optical depth, $\tau$, are generally fitted to an accuracy of a few percent. The only exception to this is for the LFI and MAP configurations, where large deviations are observed. It should however also be noted that the combined synchrotron and free-free emission "fit" done by the HFI configuration is not very well determined.

Our modelling shows, that MAP handles the twotemperature dust model equally well as the LFI concept but has some problems constraining the fit for the powerlaw dust model. Based on this a series of calculations are performed, where the dust is not fitted i.e. the $\Delta I_{\text {Dust,fit }}$ term in Eq. (6) is omitted. This was done for both the MAP and the LFI concepts, with the following results:

\section{LFI:}

\begin{tabular}{|l|c|c|}
\hline \multicolumn{3}{|c|}{ Bands [GHz]: 31.5 53 90 125 } \\
\hline Dust model: & $\left\langle\Delta T-\Delta T_{\text {fit }}\right\rangle[\mu \mathrm{K}]$ & $\sigma_{\Delta T-\Delta T_{\text {fit }}}[\mu \mathrm{K}]$ \\
\hline Power-law & 7.35 & 32.96 \\
\hline Two-temp. & 10.22 & 31.26 \\
\hline
\end{tabular}

MAP:

\begin{tabular}{|l|c|c|}
\hline \multicolumn{3}{|c|}{ Bands [GHz]: 22 30 40 60 90 } \\
\hline Dust model: & $\left\langle\Delta T-\Delta T_{\text {fit }}\right\rangle[\mu \mathrm{K}]$ & $\sigma_{\Delta T-\Delta T_{\text {fit }}}[\mu \mathrm{K}]$ \\
\hline Power-law & 10.22 & 31.26 \\
\hline Two-temp. & -0.01 & 12.75 \\
\hline
\end{tabular}

\section{Discussion}

At a first glance it is clear, that the PLANCK baseline concept will be able to extract CMBR anisotropies in the presence of contaminating foreground sources with an accuracy and a sky coverage which will allow the detection of very small temperature fluctuations in the CMBR. It can be seen, that the recovery of the anisotropies in the presence of the two-temperature dust does not cause any severe problems, except for a small systematic error seen in $\left\langle\Delta T-\Delta T_{\text {fit }}\right\rangle$. This error is most likely due to fact, that the temperature of the coldest dust component in the fitting function was chosen $1 \mathrm{~K}$ higher than the corresponding temperature in the simulated observations. The large systematic error in the LFI result for a perfect power-law dust (in the case where the dust is fitted) is most likely caused by this modelling having too few data points to constrain the fit.

It seems as if the HFI part of the mission is a very important element in its success, which can be seen from the separate analysis of the LFI and the HFI concepts. This is undoubted caused by the fact that the HFI includes the most sensitive bands around the peak of the CMBR black-body. These two bands are also included in the revised PLANCK baseline indicating that these bands are crucial to the recovery of the CMBR anisotropies.

Including IR/FIR point sources and the SunyaevZel'dovich effect in the calculations might have a negative effect on the high frequency part of the experiment, and strengthen the need for lower frequency bands in order to achieve a satisfactory anisotropy extraction. This is also an important point for further investigation. Furthermore the low frequency bands are indispensable when attempting to constrain the synchrotron radiation and especially the free-free emission by making the first all-sky maps of these galactic emission components at these frequencies, at a high level of sensitivity and with fairly high angular resolution. Even though the low frequency bands may not seem to be of great assistance in the single pixel extraction process shown above, the production of such maps would undoubted have considerable impact on the actual data processing of PLANCK observations, not to mention the astrophysical information about our Galaxy, embedded in such maps. It is important to keep in mind that the above listed results are based on the assumption that the low frequency radiation components can be fitted using power-laws. This might however not be the case and the only way to enable us to describe and remove foregrounds no matter what the sky presents to us at these frequencies, is to have complete coverage of the entire spectral range of interest.

The revised PLANCK configuration, which is largely the same concept as the baseline but with three bands omitted, perfoms almost as well as the PLANCK baseline for the reason stated above, namely that the two most sensitive bands are placed at the peak of the CMBR. The larger gaps between the frequency bands could however prove to be problematic for obtaining an accurate description of the known foreground sources and to ensure that no unknown source of confusion lurks at the frequencies 
not yet studied with high sensitivity and resolution.

The PLANCK configuration based on bolometers alone also performs almost as well as the PLANCK baseline. From a scientific point of view a pure bolometer based mission is thus clearly feasible. It is however important to keep in mind the fact, that a mission based only on detectors which require cryogenic cooling is more sensitive to technical problems, than a mission with both radiometers and bolometers. A combined mission could most likely still operate the radiometers and obtain scientific data if the cryogenic system should fail.

With respect to the MAP concept we find, that in the case where the dust is fitted, the two-temperature dust model results in a performance comparable to the LFI concept whereas the power-law dust results in a quite poorly constrained fit. The reason for this is most likely the fact that MAP only covers the $22-90 \mathrm{GHz}$ range, where dust emission gives only a small contribution to the total intensity. The attempt to fit a badly constrained foreground introduces large errors, as was also concluded by Brandt et al. (1994). With this in mind the calculations where the dust is not fitted show that MAP is doing much better when this emission component is ignored. For the power-law dust model the results obtained are comparable to those obtained using LFI, whereas the two-temperature dust scenario performs somewhat better. Under these conditions it is seen that MAP easily can deliver the intended $\frac{\Delta T}{T}$ sensitivities of $\sim 710^{-6}$ as mentioned in Sect. 2 .

The LFI concept does not seem to benefit substantially from the modelling where the dust component is not fitted. It seems as if the $125 \mathrm{GHz}$ band is stretching too far into the frequency region where the dust contribution is no longer negligible.

Since the modelling done in this study is based on the basic ideas of Brandt et al. (1994), as mentioned earlier, it is natural to compare the results of the two studies. First of all it is important to notice that Brandt et al. (1994) only carry out their calculations with very limited ranges of foreground parameters found in a $10^{\circ} \times 10^{\circ}$ patch in the sky. These parameter ranges are indicated in Fig. 2.

For the space-based experiments they examine two different frequency intervals, which can be compared to the LFI and HFI concepts, namely the range from 30 to $120 \mathrm{GHz}$ and from 150 to $435 \mathrm{GHz}$. As in this study they make 100 model calculations and fits using the same instrument and foreground characteristics in order to produce a statistical sample. They also state their results in terms of the standard deviation of the difference between the input parameters and the fitted parameters.

For both frequency ranges stated above they find $\sigma_{\Delta T-\Delta T_{\text {fit }}}$ (termed $E_{\text {rms }}$ by Brandt et al. 1994) in the order of a few $\mu \mathrm{K}$, which compares well to the results given above for the HFI concept, whereas the LFI results are a factor 10 larger. This can however be understood by looking at the noise levels assumed. In the low frequency region they operate with an noise level, which is about a factor 10 smaller than the values given for the LFI concept. For the high frequency range the assumed noise level is almost identical with the level calculated for the HFI. Generally the results obtained in this study are thus in agreement with the work done by Brandt et al. (1994).

The main result of this work is, that even extreme and poorly known foregrounds do not prevent the extraction of CMBR anisotropies with an accuracy in $\frac{\Delta T}{T} \approx 110^{-6}$. It can be very useful to search for patches with low contamination by foregrounds, but it is not imperative for the success of a CMBR anisotropy experiment like PLANCK, at least not on angular scales of about $30^{\prime}$.

Brandt et al. (1994) do not address the case of a mission covering the entire frequency range relevant for CMBR anisotropy searches but from the above considerations it seems clear, that a broad frequency coverage will allow a better extraction of the CMBR anisotropies with the intended accuracy.

Tegmark \& Efstathiou (1996) find that a pixel-by-pixel subtraction with PLANCK data yields an accuracy in the extraction of anisotropies of a few $\mu \mathrm{K}$ at a resolution of $30^{\prime}$, in good agreement with the results achieved in this study, whereas their optimal filtering technique yields results, which are about 100 times better.

Since most of the foreground parameters are rather uncertain and since they are definitely not independent of the position in the sky as discussed earlier, the above stated gain in accuracy of a factor $\sim 100$ is not directly achievable. In the reduction and analysis of data obtained with a mission like PLANCK, the very detailed all-sky maps also depicting the foregrounds could be utilized by first making a single-pixel spectral analysis as demonstrated above. One could imagine a scheme where, for each pixel, first the CMBR signal is extracted and then subtracted from the data. Then the dust parameters are extracted and this component is subtracted. Finally the synchrotron radiation and free-free emission parameters are extracted. Such an analysis would produce maps of the various foregrounds and of the CMBR with an accuracy on the $\frac{\Delta T}{T} \sim 10^{-6}$ level. These maps could then be used as the spectral and spatial templates needed to perform the optimal Wiener filtering technique to improve the accuracy in the determination of the CMBR anisotropies, thus combining the strength of both methods. The result of this combined analysis will then in the best case be the mapping of the CMBR anisotropies down to the $10^{\prime}$ scale with an accuracy of a fraction of $\frac{\Delta T}{T} \sim 10^{-6}$. Such measurements will enable us to determine most cosmological parameters to an accuracy in the order of one percent (see e.g. Jungman et al. 1996) and thus unambiguously discriminate between competing cosmological scenarios. 


\section{Conclusions}

1. Even in a worst-case foreground scenario the PLANCK baseline is able to achieve an accuracy in the measurement of CMBR fluctuations of $\frac{\Delta T}{T} \approx 110^{-6}$.

2. The dynamic range is wide, meaning that even severe limitations in our knowledge of the foreground parameters, at a given point in the sky, will not affect the above stated accuracy.

3. Increased knowledge of the foregrounds and exploitation of the spatial correlations in the foregrounds will provide even better results than those stated above. Using the results from spectral fitting as shown here could be used as input for the optimal Wiener filtering technique outlined by Tegmark \& Efstathiou (1996) to yield truly stunning results.

4. The present PLANCK frequency band baseline is optimal regarding both instrumental limitations, scientific objectives, which are not just cosmological but also include the desire to map the sky and especially the Galaxy at hitherto unobserved frequencies and finally the need of "spectral redundancy" both in case of unexpected phenomena turning up and in case of failure of one or more detectors.

Acknowledgements. We would like to thank Dr. Stephan S. Meyer from the University of Chicago, and the members of the PLANCK Science Team for fruitful discussions.

\section{References}

Alexander D.R., Ferguson J.W., 1994, in: Jørgensen U.G. (ed.) Molecules in the Stellar Environment. Lecture Notes in Physics, Vol. 428. Springer Verlag

Banday A.J., Wolfendale A.W., 1991, MNRAS 252, 462

Banday A.J., Górski K.M., Bennett C.L., et al., 1997, ApJ 475, 393

Bennett C.L., Smoot G.F., Hinshaw G., et al., 1992, ApJ 396, L7

Bennett C.L., Banday A.J., Górski K.M., et al., 1996, ApJ 464, L1

Bersanelli M., Bouchet F.R., Efstathiou G., et al., 1996, COBRAS/SAMBA. A Mission Dedicated to Imaging the Anisotropies of the Cosmic Microwave Background, Report on the Phase A Study, ESA

Boulanger F., Abergel A., Bernard J.-P., et al., 1996, A\&A 312,256

Brandt W.N., Lawrence C.R., Readhead A.C.S., Pakianathan J.N., Fiola T.M., 1994, ApJ 424, 1
Brosa U., 1994, ADAPTION Program Manual, Brosa GmbH Danese L., Toffolatti L., Franceschini A., Bersanelli M., Mandolesi N., 1996, Astroph. Lett. Comm. 33, 257

Fixsen D.J., Cheng E.S., Gales J.M., et al., 1996, ApJ 473, 576

Franceschini A., Toffolatti L., Danese L., De Zotti G., 1989, ApJ 344, 35

Górski K.M., Banday A.J., Bennett C.L., et al., 1996, ApJ 464, L11

Haslam C.G.T., Salter C.J., Stoffel H., Wilson W.E., 1982, A\&AS 47, 1

Jungman G., Kamionkowski M., Kosowsky A., Spergel D.N., 1996, Phys. Rev. D54, 1332

Knapp G.R., 1991, in: Lambert D.L. (ed.) Frontiers of Stellar Evolution, ASP Conf. Ser. 20

Kogut A., Banday A.J., Bennett C.L., et al., 1995, ApJ 460, 1

Lawson K.D., Mayer C.J., Osborne J.L., Parkinson M.L., 1987, MNRAS 225, 307

Linden-Vørnle M.J.D., 1995, The Expected Observations of the Cosmic Microwave Background Radiation Anisotropy Made by the COBRAS/SAMBA Satellite. Master thesis, Niels Bohr Institute for Astronomy, Physics and Geophysics, Astronomical Observatory, Copenhagen, Denmark

Muciaccia P.F., Bersanelli M., De Bernardis P., et al., 1996, A\&AS 117,569

Partridge R.B., 1995, 3K: The Cosmic Microwave Background Radiation. Cambridge Astrophyscis Series No. 25. Cambridge University Press

Press W.H., Flannery B.P., Teukolsky S.A., Vetterling W.T., 1989, Numerical Recipes (FORTRAN Version). Cambridge University Press

Reach W.T., Dwek E., Fixsen D.J., et al., 1995, ApJ 451, 188

Reich W., 1982, A\&AS 48, 219

Reich P., Reich W., 1986, A\&AS 63, 205

Reynolds R.J., 1992, ApJ 392, L35

Salter C.J., Brown R.L., 1988, in: Verschuur G.L. \& Kellermann K.I. (eds.) Galactic and Extragalactic Radio Astronomy, 2nd ed. A\&A Library. Springer Verlag

Sedlmayr E., 1994, in: Jørgensen U.G. (ed.) Molecules in the Stellar Environment. Lecture Notes in Physics, Vol. 428. Springer Verlag

Simonetti J.H., Dennison B., Topasna G.A., 1996, ApJ 458, L1

Sodroski, T.J., Bennett, C.L., Boggess, N.W., et al., 1994, ApJ 428,638

Spergel D.N., Hinshaw G., Bennett C.L., 1996, MAP Home Page, URL: http://map.gsfc.nasa.gov/

Tegmark M., 1996, ApJ 464, L35

Tegmark M., Efstathiou G., 1996, MNRAS 281, 1297

Wright E.L., Mather J.C., Bennett C.L., et al., 1991, ApJ 381, 200 